\title{
Systematic Review on the Controlling Nutritional Status (CONUT) Score in Patients Undergoing Esophagectomy for Esophageal Cancer
}

\author{
KOSEI TAKAGI ${ }^{1,2}$, STEFAN BUETTNER ${ }^{1}$, JAN N.M. IJZERMANS ${ }^{1}$ and BAS P.L. WIJNHOVEN ${ }^{1}$ \\ ${ }^{1}$ Department of Surgery, Erasmus MC, University Medical Center Rotterdam, Rotterdam, the Netherlands; \\ ${ }^{2}$ Department of Gastroenterological Surgery, Okayama University Graduate School of Medicine, \\ Dentistry, and Pharmaceutical Sciences, Okayama, Japan
}

\begin{abstract}
Background/Aim: The present study aimed to examine the association of the controlling nutritional status (CONUT) score with outcomes in patients undergoing esophagectomy for esophageal cancer (EC). Materials and Methods: A systematic literature review was carried out to investigate the impact of the CONUT score in EC. Next, metaanalysis of long-term outcomes was performed. Results: The search found six eligible retrospective studies, and five studies with 952 patients were included in the meta-analysis. Metaanalysis found a significant association of the CONUT score with outcomes including overall survival [hazard ratio $(H R)=2.51,95 \%$ confidence interval $(C I)=1.75-3.60$, $p<0.001]$, cancer-specific survival $(H R=2.60,95 \% C I=1.53$ $4.41, p<0.001)$, and recurrence free survival $(H R=2.08$, 95\% CI=1.39-3.12, $p<0.001)$. Conclusion: The CONUT score may be an independent predictor associated with prognosis in patients undergoing esophagectomy for EC. However, further studies are needed to clarify the association of the CONUT score with postoperative outcomes in EC patients.
\end{abstract}

Preoperative nutritional status has recently been highlighted as an important determinant to predict prognosis of cancer (1-3). The controlling nutritional status (CONUT) score is a newly developed system for nutritional screening, which is calculated by three laboratory values: plasma albumin,

This article is freely accessible online.

Correspondence to: Kosei Takagi, MD, Ph.D., Department of Surgery, Erasmus MC, University Medical Center Rotterdam, Dr. Molewaterplein 40, 3015 GD Rotterdam, the Netherlands. Tel: +31 643802627, Fax: +31 107032396, e-mail: kotakagi15@gmail.com

Key Words: Controlling nutritional status score, esophagectomy, esophageal cancer, outcome, review. plasma cholesterol and total lymphocyte count (4). Recently, the association of the CONUT score with outcomes has been demonstrated in the field of surgical oncology (5-9). Since 2016, a few studies reported on the CONUT score in patients undergoing esophagectomy for esophageal cancer (EC) (10, 11). However, most studies included small sample sizes and the prognostic significance of the CONUT score in EC has not been systematically investigated so far.

The aim of the present study was to perform a systematic review to summarize the current published evidence regarding the CONUT score in EC. In addition, metaanalyses were carried out to investigate the prognostic significance of the CONUT score in EC.

\section{Materials and Methods}

A systematic review. A systematic literature search was conducted on the $13^{\text {th }}$ of December 2019 and applied to five databases of Embase, Medline Ovid, Web of Science, Cochrane CENTRAL, and Google scholar, to find out all available articles on the CONUT score and the association with outcome in patients undergoing esophagectomy for EC. The search was limited in English articles with no restrictions on the publication year. This study reports in compliance with the Preferred Reporting Items for Systematic Reviewers and MetaAnalyses (PRISMA) guidelines (12), and is registered with the University Hospital Medical Information Network (UMIN000040112).

First, duplicate records were removed. Next, all the title and abstracts were screened to determine the eligible studies. Finally, full-text articles were assessed to meet the inclusion criteria. The following data were extracted: study information (publication year, country of the study, and study design), patient characteristic (gender, tumor stage, the CONUT score), postoperative outcomes and prognosis [overall survival (OS), cancer-specific survival (CSS), and recurrence-free survival (RFS)] in patients with EC. The primary endpoint was prognosis such as OS, CSS, and RFS. Secondary endpoint was postoperative outcome. The quality of the studies included was assessed using the Newcastle-Ottawa quality assessment scale for cohort studies, and studies with a total scores $\geq 6$ were considered as high-quality studies (13). 
Statistical analysis. Random-effects models using the R 3.5.4. (cran.r-project.org) were performed for meta-analysis in order to calculate the pooled hazard ratios (HR) with 95\% confidence interval (CI) between high CONUT and low CONUT group. In the meta-analysis, random-effects models rather than fixed-effects models were used because of anticipated heterogeneity in the definitions of disease stage, and surgical strategies across studies. Statistical heterogeneity was evaluated by the chi-squared test $\left(\chi^{2}\right)$, $I^{2}$ statistic, with $p<0.05$ considered to be statistically significant. Funnel plots were applied to evaluate presence of publication bias.

\section{Results}

The PRISMA flow chart of articles included in this review is depicted in Figure 1. After excluding duplicate articles and screening the full-text articles, six articles were included in this study $(10,11,14-17)$. The characteristics of the study included are demonstrated in Table I. All studies were singlecenter retrospective cohort studies from Japan. The quality of the studies included was evaluated as high-quality due to a total score $\geq 6$ (details shown in Table II). No obvious asymmetry was identified in Funnel plots of meta-analysis.

The CONUT score and prognosis after esophagectomy for esophageal cancer. Five studies reported data regarding prognosis in patients undergoing esophagectomy for EC. OS was assessed in four studies $(10,14,16,17)$, CSS in two studies $(14,15)$, and RFS in two studies $(10,16)$. Data regarding the association of the CONUT score with prognosis are summarized in Table III.

Toyokawa et al. (10) investigated the effect of the CONUT score on OS and RFS compared with other biomarkers including the neutrophil to lymphocyte ratio (NLR), the platelet to lymphocyte ratio (PLR), and the Glasgow Prognostic Score (GPS) in 185 patients with EC. The results found the highest areas under the curve predicting 3-year OS in the CONUT of 0.603 , followed by the NLR of 0.564 , the GPS of 0.563 and the PLR of 0.561 . Authors concluded that the CONUT score was a significant predictor of OS $(\mathrm{HR}=2.303,95 \% \mathrm{CI}=1.191-4.455, \quad p=0.013)$ and RFS $(\mathrm{HR}=2.163,95 \% \mathrm{CI}=1.139-4.109, p=0.018)$, and was superior to NLR, PLR, and GPS as a predictor of prognosis in EC.

Yoshida et al. (14) analyzed outcomes based on the CONUT score in 373 patients undergoing esophagectomy for EC. Multivariable analyses revealed that high CONUT score $(\geq 5)$ was significantly associated with poor prognosis in terms of OS $(\mathrm{HR}=3.56,95 \% \mathrm{CI}=1.714-7.390, p<0.001)$ and CSS $(\mathrm{HR}=3.41,95 \% \mathrm{CI}=1.790-6.516, p=0.046)$.

Hirahara et al. (15) evaluated the impact of the CONUT score and the NLR as a predictive factor for CSS in 148 patients undergoing esophagectomy for EC. Their multivariable analyses identified not the NLR but the CONUT score as a significant predictor for CSS $(\mathrm{HR}=1.988$, $95 \% \mathrm{CI}=1.071-3.875, p=0.029$ ).
Hikage et al. (16) explored the effects of longitudinal data of the CONUT score and the prognostic nutritional index (PNI) on survival in 141 patients undergoing neoadjuvant chemotherapy and esophagectomy for EC. The longitudinal data of these nutritional scores before neoadjuvant chemotherapy, before surgery, on 14 days after surgery and on 4 months after surgery were assessed.

Sakai et al. (17) compared the prognostic role of preoperative immunoinflammatory and nutritional measures, including the GPS, CONUT score, PNI, C-reactive proteinto-albumin ratio (CAR), NLR, and PLR, in 105 patients with EC. Their multivariable analysis found out that the CAR was a factor most associated with OS, but the CONUT score was not a significant factor $(\mathrm{HR}=1.54,95 \% \mathrm{CI}=0.54-4.41$, $p=0.42$ ).

The meta-analysis included five studies with 952 patients, as represented in Figure 2. Meta-analysis found that high CONUT score was significantly associated with worse OS $(\mathrm{HR}=2.51, \quad 95 \% \mathrm{CI}=1.75-3.60, \quad p<0.001) \quad$ without heterogeneity $\left(\mathrm{I}^{2}=0 \%, p=0.62, \mathrm{n}=804\right)$. In addition, there was an association between a high CONUT score and poor CSS $\left(\mathrm{HR}=2.60,95 \% \mathrm{CI}=1.53-4.41, p<0.001, \mathrm{I}^{2}=26 \%, p=0.25\right.$, $\mathrm{n}=521)$ and RFS $(\mathrm{HR}=2.08,95 \% \mathrm{CI}=1.39-3.12, p<0.001$, $\mathrm{I} 2=0 \%, p=0.88, \mathrm{n}=326)$.

The CONUT score and postoperative outcomes after esophagectomy for esophageal cancer. Yoshida et al. (11) investigated the association of the CONUT with short-term outcomes in EC patients. In the multivariable analysis, the CONUT score was revealed to be a significant factor associated with overall complications $(\mathrm{OR}=2.75,95 \% \mathrm{CI}=1.08$ $7.02, p=0.034)$ as well as severe complications $(\mathrm{OR}=3.07$, $95 \% \mathrm{CI}=1.002-9.43, p=0.049)$. In contrast, another study from their group (14) demonstrated no significant differences in short-term outcomes between patients evaluated for the degree of malnutrition using the CONUT score.

\section{Discussion}

This systematic review investigated the impact of the CONUT score on prognosis and complications in patients undergoing esophagectomy for EC, and summarized the current evidence regarding the prognostic role of the CONUT score in EC. The meta-analysis of five studies showed a worse long-term survival in patients with higher CONUT score. The CONUT score was associated with postoperative complications in only one study.

Esophageal cancer frequently leads to significant weight loss and malnutrition due to progressive dysphagia, resulting in poor survival (18). Moreover, esophagectomy is known as a complex surgical procedure with a high incidence of postoperative complications (19). Preoperative assessment of nutritional status as well as body composition and to 


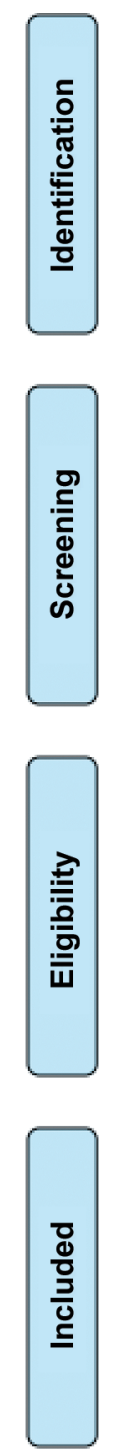

Records identified through database searching $(n=415)$
Additional records identified through other sources $(n=0)$

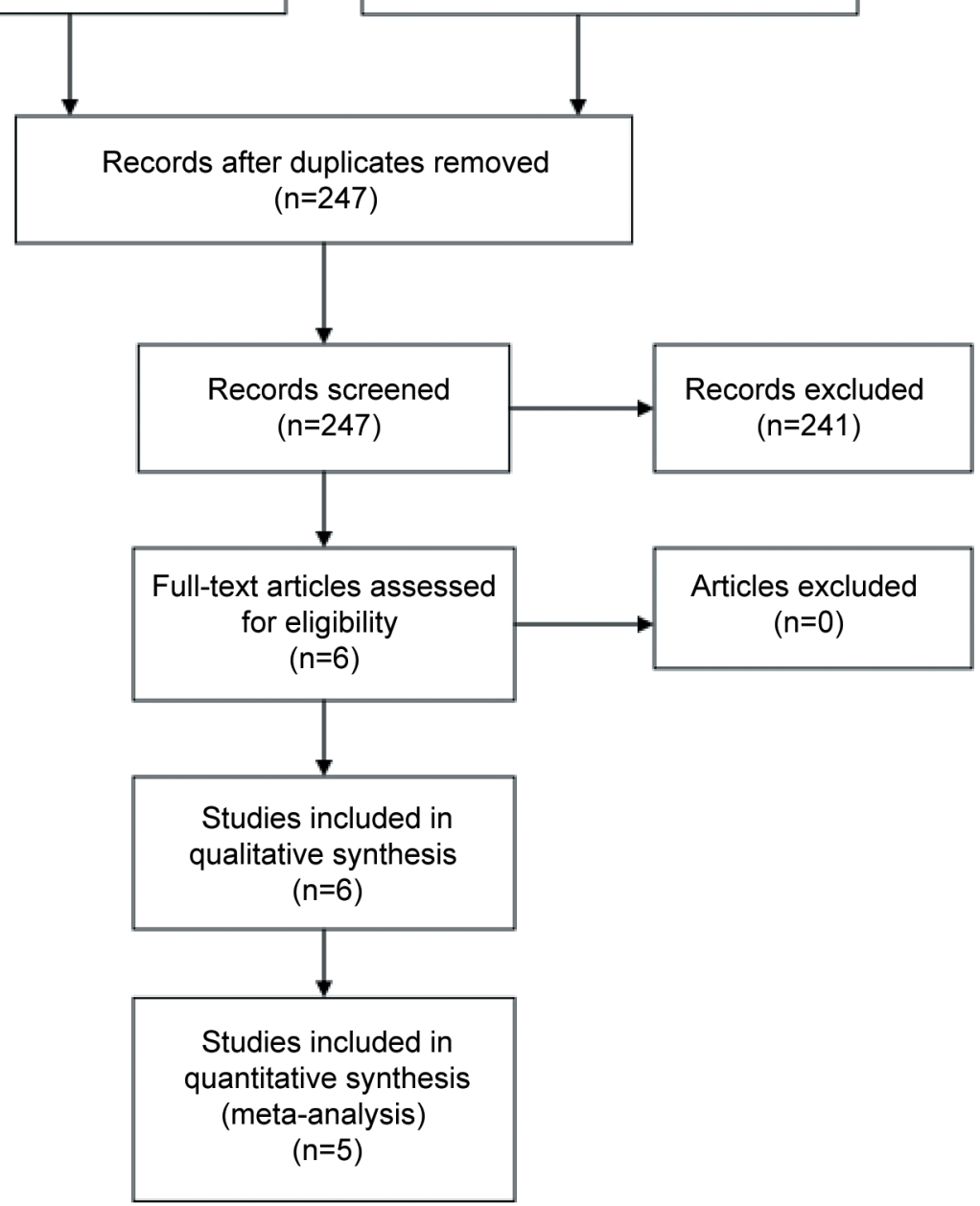

Figure 1. PRISMA 2009 flow diagram.

optimize patient's condition when indicated could be essential to improve postoperative outcomes after esophagectomy $(18,20)$. Several nutritional biomarkers have been shown to have well described relationships with longterm survival $(10,15-17)$. The PNI is one of the prognostic biomarkers demonstrated to be associated with prognosis as well as clinicopathological features in EC (21). The investigation of body composition, so called sarcopenia, in EC patients for the purpose of nutritional evaluation may be useful, as sarcopenia has been reported to be significantly associated with worse prognosis after esophagectomy (22).

A recent meta-analysis has reported the CONUT score to be associated with long-term prognosis in gastrointestinal cancers (5). However, this analysis was not specific for patients with EC and the value of the CONUT score should be analyzed separately given the different postoperative risk and cancer prognosis. Therefore, the present meta-analysis is the first to indicate the CONUT score to be an independent biomarker associated with survival in EC. Although the biological mechanism is not well understood, several studies have reported the association of each parameter in the CONUT score with prognosis in various cancers. The serum albumin is well known to reflect nutritional status as well as systematic inflammatory response, and has been reported to be associated with prognosis in cancer $(2,23)$. In addition, the total cholesterol level has been described to be related to cancer survival (24). The total lymphocyte count is known as an indicator reflecting the immunological and nutritional 
Table I. Studies on the CONUT score in patients undergoing esophagectomy for esophageal cancer.

\begin{tabular}{|c|c|c|c|c|c|c|c|c|}
\hline Study & Year & Country & $\begin{array}{l}\text { Study } \\
\text { design }\end{array}$ & $\begin{array}{c}\text { Number } \\
\text { (Male) }\end{array}$ & $\begin{array}{l}\text { Tumor } \\
\text { stage }\end{array}$ & $\begin{array}{l}\text { Cut-off for high } \\
\text { CONUT group } \\
\text { (Prevalence of high } \\
\text { CONUT score) }\end{array}$ & Treatment & $\begin{array}{l}\text { End } \\
\text { points }\end{array}$ \\
\hline Yoshida et al. (11) & 2016 & Japan & $\begin{array}{l}\text { Retrospective } \\
\text { single center }\end{array}$ & $352(314)$ & $\begin{array}{l}\text { I: } 198 \\
\text { II: } 62 \\
\text { III: } 77 \\
\text { IV: } 15\end{array}$ & $\geq 5(6.0 \%)$ & Resection & Complications \\
\hline Toyokawa et al. (10) & 2016 & Japan & $\begin{array}{l}\text { Retrospective } \\
\text { single center }\end{array}$ & $185(152)$ & $\begin{array}{c}\text { I: } 67 \\
\text { II: } 78 \\
\text { III/IV: } 40\end{array}$ & $\geq 3(9.2 \%)$ & Resection & $\begin{array}{l}\text { OS } \\
\text { RFS }\end{array}$ \\
\hline Yoshida et al. (14) & 2017 & Japan & $\begin{array}{l}\text { Retrospective } \\
\text { single center }\end{array}$ & 373 (179) & $\begin{array}{l}\text { I: } 136 \\
\text { II: } 34 \\
\text { III: } 35 \\
\text { IV: } 2\end{array}$ & $\geq 5(5.9 \%)$ & Resection & $\begin{array}{l}\text { OS } \\
\text { CSS }\end{array}$ \\
\hline Hirahara et al. (15) & 2018 & Japan & $\begin{array}{l}\text { Retrospective } \\
\text { single center }\end{array}$ & $148(132)$ & $\begin{array}{l}\text { I: } 56 \\
\text { II: } 38 \\
\text { III: } 54\end{array}$ & $\geq 2(52.7 \%)$ & Resection & CSS \\
\hline Hikage et al. (16) & 2019 & Japan & $\begin{array}{l}\text { Retrospective } \\
\text { single center }\end{array}$ & $141(116)$ & $\begin{array}{c}0: 5 \\
\text { I: } 22 \\
\text { II: } 45 \\
\text { III: } 52 \\
\text { IV: } 17\end{array}$ & $\geq 5(27 \%)$ & Resection & $\begin{array}{l}\text { OS } \\
\text { RFS }\end{array}$ \\
\hline Sakai et al. (17) & 2020 & Japan & $\begin{array}{l}\text { Retrospective } \\
\text { single center }\end{array}$ & $105(93)$ & $\begin{array}{l}\text { I: } 27 \\
\text { II: } 25 \\
\text { III: } 40 \\
\text { IV: } 13\end{array}$ & $\geq 4(8.6 \%)$ & Resection & OS \\
\hline
\end{tabular}

CONUT: Controlling nutritional status; OS: overall survival; CSS: cancer-specific survival; RFS: recurrence-free survival.

Table II. The Newcastle-Ottawa scale for quality assessment of the included studies.

\begin{tabular}{|c|c|c|c|c|c|c|c|c|c|}
\hline \multirow[t]{2}{*}{ Study } & \multicolumn{4}{|c|}{ Selection } & \multirow{2}{*}{$\begin{array}{l}\text { Comparability } \\
\text { Comparability } \\
\text { of cohorts on } \\
\text { the basis of } \\
\text { the design or } \\
\text { analysis }\end{array}$} & \multicolumn{3}{|c|}{ Outcome } & \multirow{2}{*}{$\begin{array}{l}\text { Total } \\
\text { score }\end{array}$} \\
\hline & $\begin{array}{l}\text { Representati- } \\
\text { veness of } \\
\text { the exposed } \\
\text { cohort }\end{array}$ & $\begin{array}{l}\text { Selection } \\
\text { of the } \\
\text { non-exposed } \\
\text { cohort }\end{array}$ & $\begin{array}{c}\text { Ascertainment } \\
\text { of exposure }\end{array}$ & $\begin{array}{l}\text { Demonstration } \\
\text { that outcome } \\
\text { of interest was } \\
\text { not present at } \\
\text { start of study }\end{array}$ & & $\begin{array}{l}\text { Assessment } \\
\text { of outcome }\end{array}$ & $\begin{array}{l}\text { Was follow-up } \\
\text { long enough } \\
\text { for outcomes } \\
\text { to occur }\end{array}$ & $\begin{array}{l}\text { Adequacy } \\
\text { of } \\
\text { follow-up } \\
\text { of cohorts }\end{array}$ & \\
\hline Total score & 1 & 1 & 1 & 1 & 2 & 1 & 1 & 1 & 9 \\
\hline Yoshida et al. (11) & 1 & 1 & 1 & 0 & 1 & 1 & 1 & 1 & 7 \\
\hline Toyokawa et al. (10) & 1 & 1 & 1 & 0 & 2 & 1 & 1 & 1 & 8 \\
\hline Yoshida et al. (14) & 1 & 1 & 1 & 0 & 1 & 1 & 1 & 1 & 7 \\
\hline Hirahara et al. (15) & 1 & 1 & 1 & 0 & 2 & 0 & 1 & 1 & 7 \\
\hline Hikage et al. (16) & 1 & 1 & 1 & 0 & 1 & 1 & 1 & 1 & 7 \\
\hline Sakai et al. (17) & 1 & 1 & 1 & 0 & 2 & 0 & 1 & 1 & 7 \\
\hline
\end{tabular}

status, and is also reported to be associated with prognosis in EC patients $(10,25)$. These findings suggest that the CONUT score could be a prognostic indicator of survival in EC patients. However, the evidence of the CONUT score on risks of complication and clinico-pathological parameters is still limited.
The present study has several limitations. First, the methodologic quality of the studies included in the metaanalysis should be acknowledged, such as the retrospective data with small sample sizes and different use of cut-off values of the CONUT scores in each study. Furthermore, all studies included were single-center series from Japan. 
a

\section{Study}

Toyokawa 2016

Yoshida 2017

Hikage 2019

Sakai 2020

Random effects model

Heterogeneity: $I^{2}=0 \%, \tau^{2}=0, p=0.62$ $\log \mathrm{HR}$ SE $\log \mathrm{HR}$

$0.83 \quad 0.3365$

$1.27 \quad 0.3728$

0.910 .3182

$0.43 \quad 0.5357$

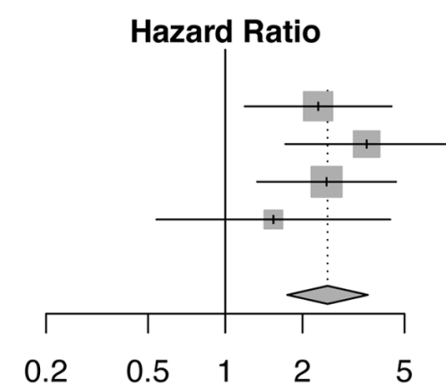

HR $95 \%-\mathrm{Cl}$ Weight

$2.30[1.19 ; 4.45] \quad 30.0 \%$

$3.56[1.71 ; 7.39] \quad 24.5 \%$

$2.48[1.33 ; 4.63] \quad 33.6 \%$

$1.54[0.54 ; 4.40] \quad 11.9 \%$

$2.51[1.75 ; 3.60] 100.0 \%$

b

\section{Study}

Yoshida 2017

Hirahara 2018

Random effects model

Heterogeneity: $I^{2}=26 \%, \tau^{2}=0.0374, p=0.25$ $\log \mathrm{HR}$ SE logHR

$1.23 \quad 0.3296$

$0.69 \quad 0.3280$

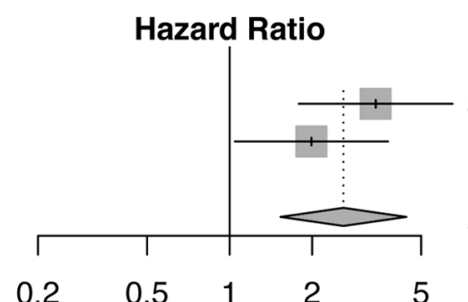

HR $95 \%-\mathrm{Cl}$ Weight

$3.41[1.79 ; 6.51] \quad 49.8 \%$ $1.99[1.05 ; 3.78] \quad 50.2 \%$

$2.60[1.53 ; 4.41] 100.0 \%$

C

\section{Study}

Toyokawa 2016

Hikage 2019

0.77
0.71

0.3273

0.2670

\section{Random effects model}

Heterogeneity: $I^{2}=0 \%, \tau^{2}=0, p=0.88$

$\log H R$ SE logHR

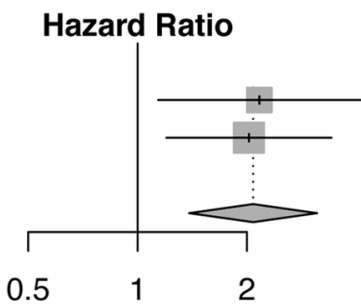

HR $\quad 95 \%-C l$ Weight

$2.16[1.14 ; 4.11] \quad 40.0 \%$ $2.02[1.20 ; 3.42] \quad 60.0 \%$

$2.08[1.39 ; 3.12] 100.0 \%$

Figure 2. Forest plots demonstrating the association of the CONUT score with (a) overall survival; (b) cancer-specific survival; and (c) recurrencefree survival.

Table III. Studies reporting the association between the CONUT score and outcomes in patients with esophageal cancer.

\begin{tabular}{|c|c|c|}
\hline Study & Postoperative outcomes & Long-term outcomes \\
\hline Yoshida et al. (11) & $\begin{array}{c}\text { Any complications: } \mathrm{OR}=2.75(1.08-7.02), p=0.034 * \\
\text { Severe complications: } \mathrm{OR}=3.07(1.002-9.43), p=0.049^{*} \\
(\mathrm{CONUT} \geq 5 \text { vs. } 0-4)\end{array}$ & n.a. \\
\hline Toyokawa et al. (10) & n.a & $\begin{aligned} \text { OS: } H R= & 2.303(1.191-4.455), p=0.013^{*} \\
\text { RFS: } H R= & 2.163(1.139-4.109), p=0.018^{*} \\
& (\text { CONUT } \geq 3 \text { vs. } 0-2)\end{aligned}$ \\
\hline Yoshida et al. (14) & n.a & $\begin{aligned} \text { OS: } \mathrm{HR}= & 3.56(1.714-7.390), \mathrm{p}<0.001^{*} \\
\mathrm{CSS}: \mathrm{HR}= & 3.41(1.790-6.516), p=0.046^{*} \\
& (\mathrm{CONUT} \geq 5 \text { vs. } 0-4)\end{aligned}$ \\
\hline Hirahara et al. (15) & n.a & CSS: HR $=1.988(1.071-3.875), p=0.029 *$ \\
\hline Hikage et al. (16) & n.a. & $\begin{array}{l}\text { OS: } H R=2.484(1.327-4.620), p=0.005^{*} \\
\text { RFS: } 1.988(1.071-3.875) \cdot p=0.008^{*} \\
\quad(C O N U T \geq 5 \text { vs. } 0-4 \text { on POD } 14)\end{array}$ \\
\hline Sakai et al. (17) & n.a & OS: $\mathrm{HR}=1.54(0.54-4.41), p=0.42 * *$ \\
\hline
\end{tabular}

OR and HR is shown with $95 \%$ confidence interval. *Multivariable analysis; **Univariate analysis. CONUT: Controlling nutritional status; OS: overall survival; CSS, cancer-specific survival; RFS: recurrence-free survival; OR: odds ratio; HR: hazard ratio; POD: postoperative day; n.a.: not available. 
Therefore, it is uncertain whether the findings can be applied to other populations. We did not perform meta-analysis for postoperative complications and clinicopathological factors due to limited available data. Finally, the comparative analysis between the CONUT score and other biomarkers was not conducted in this study. However several studies have already investigated the prognostic role of the CONUT score compared to other biomarkers including PNI, PLR, NLR, GPS, and CAR (10, 15-17). Most of the studies reported that the CONUT score was the most significant indicator to predict prognosis $(10,15,16)$. In contrast, one study showed that CAR was the best prognostic factor (17). Accordingly, further large well-designed studies should be performed to better understand the clinical role of the CONUT score on outcomes in EC patients.

\section{Conclusion}

The assessment of the CONUT score may be clinically useful to estimate prognosis in patients undergoing esophagectomy for EC. The CONUT score has the potential to be helpful to estimate the postoperative complication risk after esophagectomy. However, further prospective larger studies are necessary to clarify the association of the CONUT score with short-term outcomes and clinicopathological factors in EC patients.

\section{Conflicts of Interest}

The Authors declare no conflicts of interest regarding this study.

\section{Authors' Contributions}

K.T. contributed to the study conception and design, the acquisition of data, the development of the protocol, and the drafting of the manuscript. S.B. contributed to the analysis and interpretation of the descriptive and the revising the final draft. J.N.M.I. and B.P.L.W contributed to the development of the protocol and the critical revising of the final draft. All Authors have approved the final version.

\section{Acknowledgements}

The Authors thank Wichor M. Bramer and Sabrina Gunput (Biomedical Information Specialists) from the Medical Library in Erasmus MC, Erasmus University Medical Centre Rotterdam (Rotterdam, the Netherlands) for their involvement in the search terms.

\section{References}

1 Kyle UG, Kossovsky MP, Karsegard VL and Pichard C: Comparison of tools for nutritional assessment and screening at hospital admission: a population study. Clin Nutr 25(3): 409417, 2006. PMID: 16356595. DOI: 10.1016/j.clnu.2005.11.001
2 Sun K, Chen S, Xu J, Li G and He Y: The prognostic significance of the prognostic nutritional index in cancer: a systematic review and meta-analysis. J Cancer Res Clin Oncol 140(9): 1537-1549, 2014. PMID: 24878931. DOI: 10.1007/s00432-014-1714-3

3 Fruchtenicht AV, Poziomyck AK, Kabke GB, Loss SH, Antoniazzi JL, Steemburgo T and Moreira LF: Nutritional risk assessment in critically ill cancer patients: systematic review. Rev Bras Ter Intensiva 27(3): 274-283, 2015. PMID: 26270855. DOI: $10.5935 / 0103-507 X .20150032$

4 Ignacio de Ulíbarri J, González-Madroño A, de Villar NG, González P, González B, Mancha A, Rodríguez F and Fernández G: CONUT: a tool for controlling nutritional status. First validation in a hospital population. Nutr Hosp 20(1): 38-45, 2005. PMID: 15762418.

5 Zhang Y and Zhang X: Controlling nutritional status score, a promising prognostic marker in patients with gastrointestinal cancers after surgery: A systematic review and meta-analysis. Int J Surg 55: 39-45, 2018. PMID: 29783000. DOI: 10.1016/ j.ijsu.2018.05.018

6 Takagi K, Domagala P, Polak WG, Buettner S and Ijzermans JNM: The controlling nutritional status score and postoperative complication risk in gastrointestinal and hepatopancreatobiliary surgical oncology: A Systematic review and meta-analysis. Ann Nutr Metab 74(4): 303-312, 2019. PMID: 31013491. DOI: $10.1159 / 000500233$

7 Takagi K, Domagala P, Polak WG, Buettner S and Ijzermans JNM: Prognostic significance of the controlling nutritional status (CONUT) score in patients undergoing hepatectomy for hepatocellular carcinoma: a systematic review and metaanalysis. BMC Gastroenterol 19(1): 211, 2019. PMID: 31818259. DOI: 10.1186/s12876-019-1126-6

8 Takagi K, Domagala P, Polak WG, Buettner S, Wijnhoven BPL and Ijzermans JNM: Prognostic significance of the controlling nutritional status (CONUT) score in patients undergoing gastrectomy for gastric cancer: a systematic review and metaanalysis. BMC Surg 19(1): 129, 2019. PMID: 31488105. DOI: 10.1186/s12893-019-0593-6

9 Takagi K, Buettner S and Ijzermans JNM: Prognostic significance of the controlling nutritional status (CONUT) score in patients with colorectal cancer: A systematic review and metaanalysis. Int J Surg 78: 91-96, 2020. PMID: 32335238. DOI: 10.1016/j.ijsu.2020.04.046

10 Toyokawa T, Kubo N, Tamura T, Sakurai K, Amano R, Tanaka $\mathrm{H}$, Muguruma K, Yashiro M, Hirakawa K and Ohira M: The pretreatment Controlling Nutritional Status (CONUT) score is an independent prognostic factor in patients with resectable thoracic esophageal squamous cell carcinoma: results from a retrospective study. BMC Cancer 16(1): 722, 2016. PMID: 27599460. DOI: $10.1186 / \mathrm{s} 12885-016-2696-0$

11 Yoshida N, Baba Y, Shigaki H, Harada K, Iwatsuki M, Kurashige J, Sakamoto Y, Miyamoto Y, Ishimoto T, Kosumi K, Tokunaga R, Imamura $\mathrm{Y}$, Ida $\mathrm{S}$, Hiyoshi $\mathrm{Y}$, Watanabe $\mathrm{M}$ and Baba $\mathrm{H}$ : Preoperative nutritional assessment by Controlling Nutritional Status (CONUT) is useful to estimate postoperative morbidity after esophagectomy for esophageal cancer. World J Surg 40(8): 19107, 2016. PMID: 27220507. DOI: 10.1007/s00268-016-3549-3

12 Moher D, Liberati A, Tetzlaff J and Altman DG: Preferred reporting items for systematic reviews and meta-analyses: the PRISMA statement. BMJ 339: b2535, 2009. PMID: 19622551. DOI: $10.1136 / \mathrm{bmj} . \mathrm{b} 2535$ 
13 Wells G SB, O’Connell D, Peterson J, Welch V. Wells G, Shea B, O'Connell D, Peterson J, Welch V, Losos M and Tugwell P: The Newcastle-Ottawa Scale (NOS) for assessing the quality of nonrandomized studies in meta-analysis. Available at: www.ohri.ca/programs/clinical_epidemiology/oxford.asp [Last Accessed Aug 2, 2020]

14 Yoshida N, Harada K, Baba Y, Kosumi K, Iwatsuki M, Kinoshita K, Nakamura K, Sakamoto Y, Miyamoto Y, Karashima R, Mima K, Sawayama H, Ohuchi M, Chikamoto A, Imamura Y, Watanabe $\mathrm{M}$ and Baba $\mathrm{H}$ : Preoperative controlling nutritional status (CONUT) is useful to estimate the prognosis after esophagectomy for esophageal cancer. Langenbecks Arch Surg 402(2): 333-341, 2017. PMID: 28138759. DOI: 10.1007/s00423017-1553-1

15 Hirahara N, Matsubara T, Hayashi H, Takai K, Nakada S and Tajima Y: Prognostic importance of controlling nutritional status in patients undergoing curative thoracoscopic esophagectomy for esophageal cancer. Am J Ther 25(5): e524-e32, 2018. PMID: 26866437. DOI: 10.1097/MJT.0000000000000414

16 Hikage M, Taniyama Y, Sakurai T, Sato C, Takaya K, Okamoto $\mathrm{H}$, Konno T, Ujiie N, Naitoh T, Unno $M$ and Kamei T: The influence of the perioperative nutritional status on the survival outcomes for esophageal cancer patients with neoadjuvant chemotherapy. Ann Surg Oncol 26(13): 4744-4753, 2019. PMID: 31440925. DOI: $10.1245 / \mathrm{s} 10434-019-07742-9$

17 Sakai M, Sohda M, Saito H, Ubukata Y, Nakazawa N, Kuriyama K, Hara K, Sano A, Ogata K, Yokobori T, Shirabe K and Saeki $\mathrm{H}$ : Comparative analysis of immunoinflammatory and nutritional measures in surgically resected esophageal cancer: a singlecenter retrospective study. In Vivo 34(2): 881-87, 2020. PMID: 32111799. DOI: 10.21873/invivo.11853

18 Steenhagen E, van Vulpen JK, van Hillegersberg R, May AM and Siersema PD: Nutrition in peri-operative esophageal cancer management. Expert Rev Gastroenterol Hepatol 11(7): 663-672, 2017. PMID: 28454509. DOI: 10.1080/17474124.2017.1325320

19 Ohkura Y, Miyata H, Konno H, Udagawa H, Ueno M, Shindoh J, Kumamaru H, Wakabayashi G, Gotoh M and Mori M: Development of a model predicting the risk of eight major postoperative complications after esophagectomy based on 10 826 cases in the Japan National Clinical Database. J Surg Oncol, 2019. PMID: 31823377 . DOI: $10.1002 /$ jso. 25800
20 Grotenhuis BA, Wijnhoven BP, Grüne F, van Bommel J, Tilanus HW and van Lanschot JJ: Preoperative risk assessment and prevention of complications in patients with esophageal cancer. J Surg Oncol 101(3): 270-278, 2010. PMID: 20082349. DOI: $10.1002 /$ jso. 21471

21 Xue Y, Zhou X, Xue L, Zhou R and Luo J: The role of pretreatment prognostic nutritional index in esophageal cancer: A meta-analysis. J Cell Physiol 234(11): 19655-19662, 2019. PMID: 31344989. DOI: 10.1002/jcp.28565

22 Boshier PR, Heneghan R, Markar SR, Baracos VE and Low DE: Assessment of body composition and sarcopenia in patients with esophageal cancer: a systematic review and meta-analysis. Dis Esophagus 31(8), 2018. PMID: 29846548. DOI: $10.1093 /$ dote/doy047

23 McMillan DC, Elahi MM, Sattar N, Angerson WJ, Johnstone J and McArdle CS: Measurement of the systemic inflammatory response predicts cancer-specific and non-cancer survival in patients with cancer. Nutr Cancer 41(1-2): 64-69, 2001. PMID: 12094630. DOI: 10.1080/01635581.2001.9680613

24 Zhou P, Li B, Liu B, Chen T and Xiao J: Prognostic role of serum total cholesterol and high-density lipoprotein cholesterol in cancer survivors: A systematic review and meta-analysis. Clin Chim Acta 477: 94-104, 2018. PMID: 29223765. DOI: 10.1016/j.cca.2017.11.039

25 Feng JF, Liu JS and Huang Y: Lymphopenia predicts poor prognosis in patients with esophageal squamous cell carcinoma. Medicine (Baltimore) 93(27): e257, 2014. PMID: 25501097. DOI: $10.1097 / \mathrm{MD} .0000000000000257$
Received July 22, 2020

Revised August 3, 2020

Accepted August 4, 2020 